\title{
Patterns of physical activity
} and their relationship with depression among community-dwelling older adults in Shanghai, China: a latent class approach

Yan Liang ${ }^{1 \dagger}$, Xinghui $\mathrm{Li}^{2 \dagger}$, Tingting Yang ${ }^{2}$, Mengying $\mathrm{Li}^{2}$, Ye Ruan ${ }^{3}$, Yinghua Yang ${ }^{4}$, Yanyan Huang ${ }^{5,6}$, Yihua Jiang ${ }^{7,8^{*}}$ and Ying Wang ${ }^{2, *^{*}}$

\begin{abstract}
Background: Few studies have explored patterns of physical activity (PA) and examined their relationship with depression among community-dwelling older adults. We aimed to identify the patterns of PA through a person-centered analytical approach and examine the association between quantity and patterns of PA, and depression among community-dwelling older adults.
\end{abstract}

Methods: We conducted a cross-sectional survey study in the Minhang district, Shanghai, China, in August 2019, and used a self-administered questionnaire to collect data through home visits. The total sample included 2525 older adults. This study used the Physical Activity Scale for the Elderly (PASE) to assess the quantity of PA in older adults. Depression was evaluated with the Geriatric Depression Scale (GDS). Latent class analysis (LCA) was used to identify subpopulations by shared item response patterns. Logistic regressions were performed to estimate the relationship between PASE score, patterns of PA, and depression. An exploratory analysis of joint levels and patterns of PA effects on depression was based on sample subgroups with combinations of levels and patterns of PA. Logistic regression was used to calculate the odds ratio for combined subgroups.

Results: Four latent classes were identified: "domestic types," "athletic types," "gardening/caring types," and "walkers." PASE scores and patterns of PA both were associated with depression. Older adults who were the most active (PASE quartile: $75-100 \%)$ and the athletic types had the strongest significant association with depression $(O R=0.19,95 \% \mathrm{Cl}$ : $0.06-0.65)$, followed by those who were the most active (PASE quartile: $75-100 \%)$ and the walkers $(O R=0.28,95 \% \mathrm{Cl}$ : 0.14-0.57) when compared with older adults with the least activity (PASE quartile: 0-25\%) and domestic types.

Conclusion: This study suggests both the quantity and patterns of physical activity are associated with depressive symptoms among community-dwelling older adults. Population-level intervention should encourage communitydwelling older adults to increase their quantity of PA to reduce the risk of depression. Athletics and walkers are

\footnotetext{
*Correspondence: mhjswszx@126.com; wangying1013@fudan.edu.cn

†Yan Liang and Xinghui Li contributed equally to this work.

${ }^{8}$ Minhang Branch, School of Public Health, Fudan University, 130 DongAn

Road, Shanghai 200032, China

${ }^{9}$ Key Laboratory of Health Technology Assessment, National Health

and Family Planning Commission of the People's Republic of China,

Fudan University, 130 DongAn Road, Shanghai 200032, China

Full list of author information is available at the end of the article
}

(C) The Author(s) 2021. Open Access This article is licensed under a Creative Commons Attribution 4.0 International License, which permits use, sharing, adaptation, distribution and reproduction in any medium or format, as long as you give appropriate credit to the original author(s) and the source, provide a link to the Creative Commons licence, and indicate if changes were made. The images or other third party material in this article are included in the article's Creative Commons licence, unless indicated otherwise in a credit line to the material. If material is not included in the article's Creative Commons licence and your intended use is not permitted by statutory regulation or exceeds the permitted use, you will need to obtain permission directly from the copyright holder. To view a copy of this licence, visit http://creativecommons.org/licenses/by/4.0/. The Creative Commons Public Domain Dedication waiver (http://creativecommons.org/publicdomain/zero/1.0/) applies to the data made available in this article, unless otherwise stated in a credit line to the data. 
recommended. To develop individual-level tailored interventions, more attention should be paid to older adults who are highly engaged in gardening/caring for others.

Keywords: Physical activity, Patterns, Depression, Latent class analysis, LCA, Older adults

\section{Background}

Depression is one of the most prevalent mental disorders in later life that has a high risk of disability worldwide [1-3]. Depression and depressive symptoms are attracting considerable interests due to their related consequences, including increased chronic disease [4], suicide and non-suicide mortality [5], and high disease burden [6].

Physical activity (PA) may be beneficial in reducing the risk of depression, but studies have reported mixed results [7-14]. The intensity, frequency, duration, volume, and types of physical activity may contribute to the inconsistency [15]. Previous studies have focused on the quantity or levels of PA and their relationship with depression in older people [16, 17]. Some studies showed that PA with higher frequency or moderateto-vigorous PA were associated with lower odds of depression [18, 19]; some suggested that light physical activity or lower frequency were protective [20, 21]. Recent research has shown that different patterns of PA may have an influence on depression, as some patterns of PA may be protective against depression for older adults while others may not [22, 23]. For example, daily purposeful exercise (walking, tai chi, aerobic/ strength training) showed a stronger relationship with depression than domestic or transportation-related PA $[8,9,22,23]$. Patterns of PA can provide more comprehensive information than the quantity or levels of PA. Research showed that high levels of PA across multiple domains, or athletic pattern were at lower risk for depression [22]. A key unanswered question is whether certain patterns of PA are particularly associated with decreased risk of depression at certain levels of PA. Therefore, knowledge about the joint effects of patterns of PA and levels of PA on depression risk would be helpful.

Patterns of PA include work-related (e.g. work for pay or as a volunteer), domestic-related (e.g. housework), and leisure-time activity (e.g. walking outside home) [24]. Previous studies in Western countries showed that leisure-time PA had a stronger inverse relationship with depression than other patterns of PA [22-24]. It is important to understand the contribution of specific patterns of PA on depression so as to develop appropriate public health recommendations. The association between patterns of PA and depression depends largely on the specific cultural contexts [25]. East Asians tend to take fewer physical activities and at lower intensity than those in Western countries [26], and may present distinctive patterns of PA.

It is challenging to compare the benefits of different patterns of PA as they are highly correlated; for example, older adults who do domestic work may be more likely to garden [22]. Latent class analysis (LCA) provides a person-centered approach for identification of patterns of PA and has been used in several studies [27-29]. To our knowledge, there has been no study exploring patterns of PA using LCA and examining the relationship between patterns of PA and depression among Chinese community-dwelling older adults. Our study may provide implications for developing community interventions in Eastern culture, both to support some patterns of PA and to target specific latent classes of communitydwelling older adults.

The purposes of this study were to: (1) identify the patterns of PA among Chinese community-dwelling older adults; (2) examine the independent contribution that quantity and patterns of PA have on depression among Chinese community-dwelling older adults; and (3) explore the joint effects of levels and patterns of PA on depression among Chinese community-dwelling older adults.

\section{Methods \\ Participants}

A cross-sectional survey study was conducted in the Minhang district, Shanghai, China, in August 2019. We used a self-administered questionnaire to collect data through home visits. The survey was performed by trained investigators and data were collected through face-to-face interview. The sample was randomly selected from the census database of older adults in the Minhang district, Shanghai. A stratified cluster random sampling design was used and four communities were selected based on geographical area, sex, and age. Participants were recruited with the annual health check up programs. Inclusion criteria were: 1 ) age $\geq 60$; 2) being community-dwelling; and 3) being able to communicate and willing to consent and participate. Exclusion criteria were as follows: 1 ) inability to understand and follow the assessment protocol of the study; and 2) having major neurocognitive disorders, such as dementia (confirmed by community doctors based on the health information). The total sample included 2525 older adults (see 


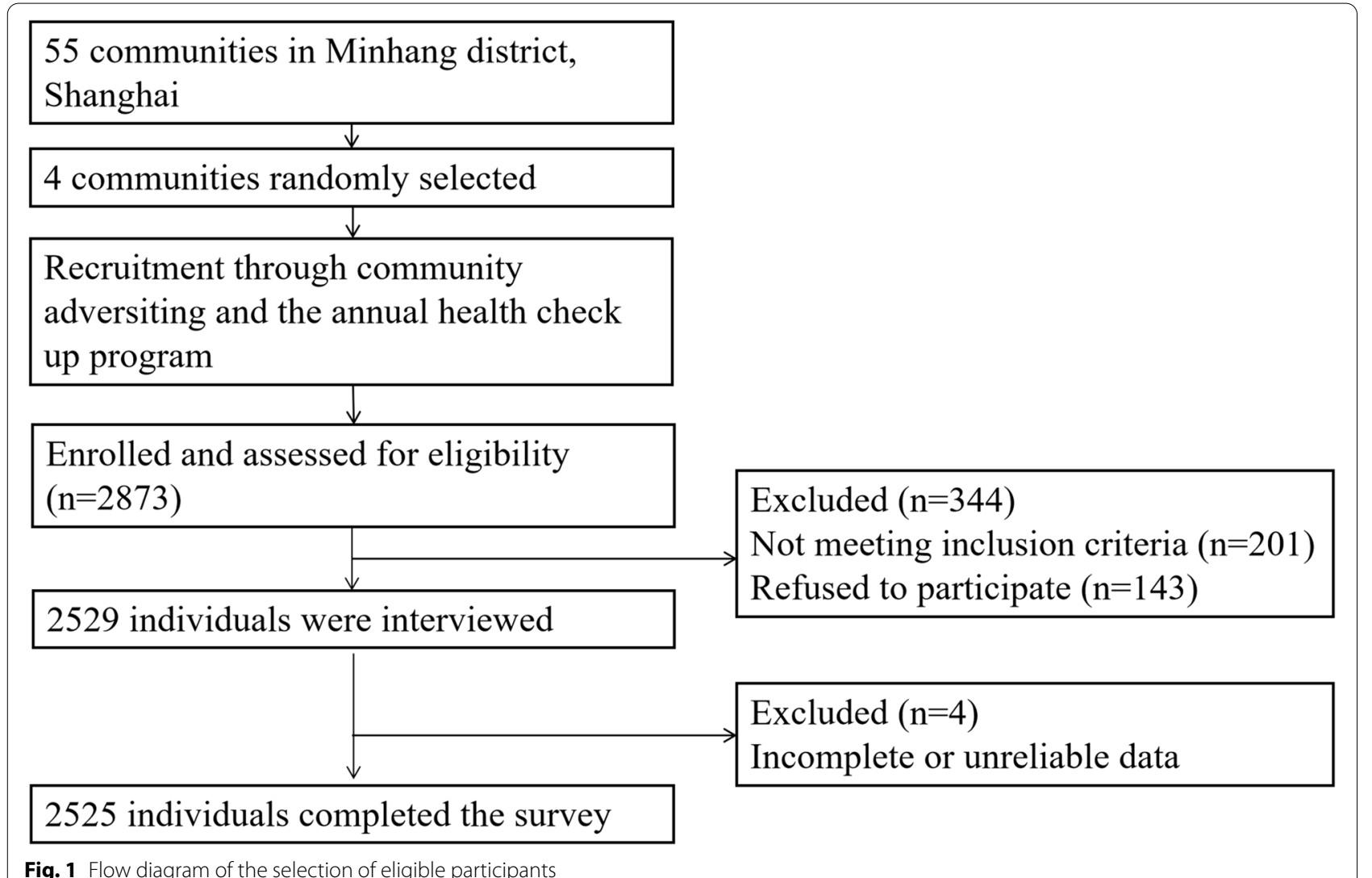

Fig. 1 Flow diagram of the selection of eligible participants

Fig. 1). This research protocol was approved by the Ethical Review Board of Fudan University (reference number: IRB\#TYSQ 2019-2-03), and informed written consent was obtained before data collection.

\section{Measures \\ Physical activity}

This study used the Physical Activity Scale for the Elderly (PASE) to assess the quantity of PA in older adults. All participants were asked to recall past-week leisure time activity, household activity, and paid or volunteer work. Items included engagement in walking, sports and recreation, muscle-strengthening and endurance exercises, housework, home repair, gardening, yard work, caring for others, and paid or volunteer work [30]. For leisure time activity, individuals responded "never," "rarely," "sometimes," or "often." The average time spent on each type of activity per day was also recorded. For household activity and paid or volunteer work, participants responded "yes" or "no." Information on the hours and types of work involved was also gathered. Each PASE item had a weight value determined by the original authors of the measurement tool [31]. The overall PASE score was calculated by multiplying the amount of time spent and participation by the PASE weight value, and then summing for a total score. The PASE scores ranged from 0 to 500 or more. The higher the scores, the higher the PA levels. PASE scores have been validated against several objective activity measures, including accelerometers and doubly labeled water [32, 33]. There is evidence supporting the validity and reliability of the Chinese version of the PASE [34].

Indicators of activity types were developed from PASE items and two modifications were made based on the study of Mooney et al. [35]. We excluded an item assessing physical activity from employment as this population were mostly retired. First, to retain information regarding activity duration in categorical variables used by LCA, we recoded PASE items indicating the duration of activity (walking, sports and recreation, and muscle-strengthening exercises) into dichotomous variables indicating ever having engaged ( $>0 \mathrm{~min} /$ day on any day) in the activity and often engaging ( $>30 \mathrm{~min} /$ day on average). Second, because many subjects reported participation in a major sport/recreation activity, exploratory LCAs that using the original separately coded items resulted in patterns of PA that were strongly influenced by intensity of a single sport/recreation activity rather than an overall pattern 
of activity. We combined all sport/recreation categories for analysis, so as to increase homogeneity of the classes and optimize interpretability of the patterns. Sports or recreational activities such as dancing, hiking, and jogging were classified as sports, and muscular strength or endurance exercises (such as sit-ups and weight lifting) were classified as exercise. Finally, a total of 12 items were used in the LCA model including the following items: ever does sports, ever exercises, ever walks, often-sports, often-exercises, often-walks, light housework, heavy housework, home repairs, yard care, outdoor gardening, and caring for others.

\section{Depressive symptoms}

The 30-item Geriatric Depression Scale (GDS), used worldwide, was utilized to assess the depressive symptoms of the participants [36]. Participants were asked to respond "yes" or "no" to each item. Summary scores ranged from 0 to 30 . A well-validated cutoff point $\geq 11$ was used to define depression [37]. The validity and reliability of the GDS-30 has been tested in China and also used in Chinese older adults [38].

\section{Sociodemographic factors and physical health status}

Participants' sociodemographic characteristics were measured as follows (see the additional file 1): (1) age (categorized as $60-74=1,75-84=2, \geq 85=3$ ); (2) sex (male $=0$, female $=1$ ); (3) marital status (married $=1$, others $=0$ ); (4) educational background (illiteracy $=1$, primary school $=2$, middle school $=3$, high school $=4$, college and more $=5)$; (5) income $(\leq 2000=1,2001$ $5000=2, \geq 5001=3$ ); and (6) living arrangements (living alone $=1$, live with spouse only $=2$, live with spouse and children $=3$, live with others $=4$ ).

Physical health status was assessed by two variables (see the additional file 1): (1) having any chronic disease (Yes $=1, \mathrm{No}_{0}$ ), and (2) self-rated health. To measure self-rated health, each participant was asked, "How would you describe your current health status?" The responses ranged from 1 to 5 indicating excellent to poor health. We reverse coded self-rated health to make higher values indicate better health so that the results were easier to interpret.

\section{Statistical analysis}

Descriptive statistics were used to summarize sample characteristics. LCA was used to identify subpopulations (latent classes) by shared item response patterns. LCA was conducted using the maximum likelihood estimation with robust standard errors. A series of LCA models with a successive number of classes were specified and selection of the optimal model that combines goodness of fit and parsimony was based on conceptual considerations and various statistical fit indices [39]. Logistic regressions were performed to estimate the relationship between PASE score, patterns of PA, and depression. In multivariate analyses, levels and patterns of PA were included simultaneously in the same model to estimate their independent contributions to depression risk. All models were adjusted for age, sex, education, income, living arrangements, self-rated health, and chronic disease. An exploratory analysis of joint levels and patterns of PA effects on depression was based on sample subgroups with combinations of levels and patterns of PA. Logistic regression was used to calculate the odds ratio for combined subgroups. LCA models were conducted in Mplus version 8.0 and all subsequent analyses were performed using Stata SE version 15.1(StataCorp., College Station, TX, USA).

\section{Results}

\section{Participant characteristics}

Table 1 presents the participants' characteristics and group differences among four patterns of PA. The majority of the sample was age $60-74$ years $(N=1829,72.4 \%)$, $55.8 \%(N=1410)$ were female, and $83.6 \%(N=2111)$ were married. The average PASE score was 119.34 $(\mathrm{SD}=41.93)$. A minority $(13.4 \%)$ reported depression. Significant differences were found among groups of four patterns of PA in all characteristics expect income.

\section{Patterns of physical activity: a four-class model}

Table 2 presents results from the LCA. Five latent class models were created, specifying latent class counts from two to six. According to recommendations of model selection in LCA [40, 41], we chose a four-class solution as the best fitting model. In a large sample, the sample-size adjusted BIC (aBIC) will keep decreasing with the increase of latent class counts [42]. But a fourclass model showed the highest entropy, representing the highest certainty of classification. Moreover, the four-class model was interpretable and reasonably well defined (Fig. 2). The final latent classes were as follows: 1) older adults who reported housework but little other activities (29.7\% of participants, "domestic types"); 2) older adults who were physically active, especially engaging in sports (11.7\% of participants, "athletic types"); 3 ) older adults who reported yard care, gardening and caring for others but little other activities (20.9\% of participants, "gardening/caring types"); and 4) older adults who reported walking and some housework (37.7\% of participants, "walkers"). Fig. 2 presents the proportion of physical activities engagement of community-dwelling older adults in the condition of latent class assignment. 
Table 1 Participants characteristics and group differences among four patterns of PA $(N=2525)$

\begin{tabular}{|c|c|c|c|c|c|c|}
\hline Characteristics & $\begin{array}{l}\text { Whole } \\
\text { sample } \\
(n=2525) \\
\mathrm{n}(\%) \text { or } \\
\text { mean } \pm S D\end{array}$ & $\begin{array}{l}\text { Domestic types } \\
(n=751) \\
\mathrm{n}(\%) \text { or mean } \pm S D\end{array}$ & $\begin{array}{l}\text { Athletic types }(n=295) \\
\mathrm{n}(\%) \text { or mean } \pm S D\end{array}$ & $\begin{array}{l}\text { Gardening/Caring } \\
\text { types }(n=528) \\
\mathrm{n}(\%) \text { or mean } \pm S D\end{array}$ & $\begin{array}{l}\text { Walkers }(n=951) \\
\mathrm{n}(\%) \text { or mean } \pm S D\end{array}$ & $P$ Value \\
\hline Age group (years) & & & & & & $<0.001$ \\
\hline $60-74$ & $1829(72.4)$ & $575(76.6)$ & $235(79.7)$ & $227(43.0)$ & $792(83.3)$ & \\
\hline $75-84$ & $503(19.9)$ & $147(19.6)$ & $55(18.6)$ & $169(32.0)$ & $132(13.9)$ & \\
\hline$\geq 85$ & $193(7.7)$ & $29(3.9)$ & $5(1.7)$ & $132(25.0)$ & $27(2.8)$ & \\
\hline Gender & & & & & & $<0.001$ \\
\hline Male & $1115(44.2)$ & $296(39.4)$ & $122(41.4)$ & $270(51.1)$ & $427(44.9)$ & \\
\hline Female & $1410(55.8)$ & $455(60.6)$ & $173(58.6)$ & $258(48.9)$ & $524(55.1)$ & \\
\hline Education & & & & & & $<0.001$ \\
\hline Illiteracy & $194(7.7)$ & $44(5.9)$ & $11(3.7)$ & $81(15.3)$ & $58(6.1)$ & \\
\hline Primary school & $463(18.3)$ & $142(18.9)$ & $34(11.5)$ & $119(22.5)$ & $168(17.7)$ & \\
\hline Middle school & $901(35.7)$ & $282(37.5)$ & $112(38.0)$ & $153(29.0)$ & $354(37.2)$ & \\
\hline Highschool & $658(26.1)$ & $191(25.4)$ & $89(30.2)$ & $115(21.8)$ & $263(27.7)$ & \\
\hline College and more & $309(12.2)$ & $92(12.3)$ & 49 (16.6) & $60(11.4)$ & $108(11.4)$ & \\
\hline Marital status & & & & & & $<0.001$ \\
\hline Married & 2111 (83.6) & $623(83.0)$ & $260(88.1)$ & $394(74.6)$ & $834(87.7)$ & \\
\hline Others & $414(16.4)$ & $128(17.0)$ & $35(11.9)$ & $134(25.4)$ & $117(12.3)$ & \\
\hline Income (Yuan) & & & & & & 0.014 \\
\hline$\leq 2000$ & $327(12.9)$ & $109(14.5)$ & $20(6.8)$ & $71(13.5)$ & $127(13.3)$ & \\
\hline $2001-5000$ & $1757(69.6)$ & $503(67.0)$ & $231(78.3)$ & $363(68.8)$ & $660(69.4)$ & \\
\hline$\geq 5001$ & $441(17.5)$ & $139(18.5)$ & $44(14.9)$ & $94(17.8)$ & $164(17.3)$ & \\
\hline Living arrangements & & & & & & $<0.001$ \\
\hline Living alone & $199(7.9)$ & $75(10.0)$ & $18(6.1)$ & $47(8.9)$ & $59(6.2)$ & \\
\hline Live with spouse only & $1381(54.7)$ & $385(51.3)$ & $180(61.0)$ & $269(51.0)$ & $547(57.5)$ & \\
\hline $\begin{array}{l}\text { Live with spouse and } \\
\text { children }\end{array}$ & $459(18.2)$ & $140(18.6)$ & $53(18.0)$ & $83(15.7)$ & $183(19.2)$ & \\
\hline Live with others & $486(19.2)$ & $151(20.1)$ & $44(14.9)$ & $129(24.4)$ & $162(17.0)$ & \\
\hline Self-rated health & $2.90 \pm 0.99$ & $2.95 \pm 0.98$ & $3.13 \pm 0.96$ & $2.43 \pm 0.96$ & $3.05 \pm 0.93$ & $<0.001$ \\
\hline Chronic disease & & & & & & $<0.001$ \\
\hline Yes & $1652(65.4)$ & $493(65.7)$ & $169(57.3)$ & $412(78.0)$ & $578(60.8)$ & \\
\hline No & $873(34.6)$ & $258(34.3)$ & $126(42.7)$ & $116(22.0)$ & $373(39.2)$ & \\
\hline PASE score & $119.3 \pm 41.9$ & $93.6 \pm 31.9$ & $144.5 \pm 60.2$ & $132.0 \pm 27.4$ & $124.8 \pm 38.6$ & $<0.001$ \\
\hline PASE quartile & & & & & & $<0.001$ \\
\hline $0-25 \%$ & $642(25.4)$ & $433(57.7)$ & $28(9.5)$ & $18(3.4)$ & $163(17.1)$ & \\
\hline $25-50 \%$ & $627(24.8)$ & $154(20.5)$ & $58(19.7)$ & 98 (18.6) & $317(33.3)$ & \\
\hline $50-75 \%$ & $631(25.0)$ & $109(14.5)$ & $86(29.1)$ & $232(43.9)$ & $204(21.5)$ & \\
\hline $75-100 \%$ & $625(24.8)$ & $55(7.3)$ & $123(41.7)$ & $180(34.1)$ & $267(28.1)$ & \\
\hline GDS score & & & & & & $<0.001$ \\
\hline$<11$ & 2186 (86.6) & $651(86.7)$ & 285 (96.6) & $386(73.1)$ & $864(90.8)$ & \\
\hline$\geq 11$ & $339(13.4)$ & $100(13.3)$ & $10(3.4)$ & $142(26.9)$ & $87(9.2)$ & \\
\hline
\end{tabular}

Notes: One-way ANOVA was used for continuous variables, and chi-square test was used for categorical variables to explore differences among four patterns of PA: domestic types, athletic types, gardening/caring types, and walkers. One-way ANOVA Bonferroni correction post hoc tests are significant if $\mathrm{p}<0.05$ (correction already included). Chi-square post hoc bivariate tests are significant if $p<0.0125$ (Bonferroni correction)

Relationship between PASE scores, patterns of PA, and depression

Patterns of PA were associated with depression: $26.9 \%$ of the gardening/caring class was depressed, compared with
$13.3,3.4$, and $9.2 \%$ of the domestic types, athletic types, and walkers, respectively ( $\chi^{2}$ test, $\left.p<0.001\right)$. In multivariable models (Table 3), PASE scores and patterns of PA both were associated with depression, suggesting that 
Table 2 LCA model fit statistics

\begin{tabular}{lllllll}
\hline Classes & AIC & BIC & aBIC & Entropy & LMR & BLRT \\
\hline 2 & $24,642.702$ & $24,788.552$ & $24,709.121$ & 0.883 & $<0.0001$ & $<0.0001$ \\
3 & $23,673.848$ & $23,895.54$ & $23,774.804$ & 0.895 & $<0.0001$ & $<0.0001$ \\
4 & $23,368.236$ & $23,665.77$ & $23,503.729$ & 0.915 & $<0.0001$ & $<0.0001$ \\
5 & $23,166.994$ & $23,540.37$ & $23,337.025$ & 0.868 & $<0.0001$ & $<0.0001$ \\
6 & $23,021.524$ & $23,470.741$ & $23,226.092$ & 0.784 & 0.3153 & $<0.0001$ \\
\hline
\end{tabular}

Note: AIC, Akaike information criteria; BIC, Bayesian information criteria; aBIC, sample-size adjusted BIC; LMR, $p$-value for the Lo-Mendell-Rubin likelihood ratio test; BLRT, $p$-value for the bootstrapped likelihood ratio test

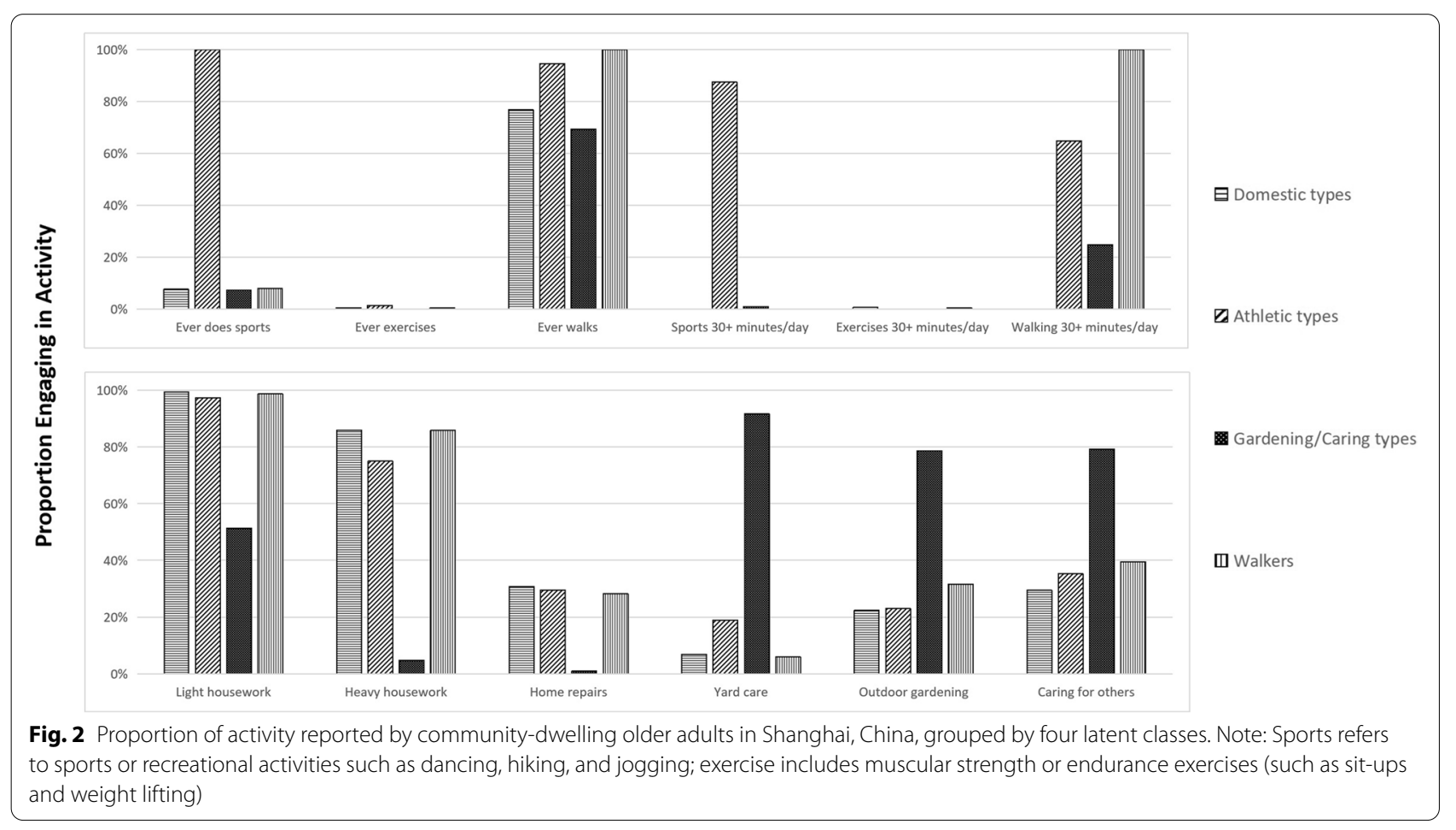

Table 3 Logistic regression models showing the associations between PASE score, patterns of PA, and depression $(n=2525)$

\begin{tabular}{llll}
\hline & Model 1 & Model 2 & Model 3 \\
\hline Variable & OR $(95 \% \mathrm{Cl})$ & OR $(95 \% \mathrm{Cl})$ & $\mathrm{OR}(95 \% \mathrm{Cl})$ \\
Standardized PASE score & $0.77^{* *}(0.66-0.91)$ & & $0.78^{*}(0.64-0.94)$ \\
Patterns of PA (Domestic types as reference) & & & $0.35^{* *}(0.17-0.71)$ \\
$\quad$ Athletic types & & $0.27^{* * *}(0.14-0.53)$ & $1.55^{*}(1.06-2.26)$ \\
$\quad$ Gardening/Caring types & & $1.25(0.89-1.75)$ & $0.88(0.62-1.24)$ \\
$\quad$ Walkers & & $0.73(0.53-1.01)$ & \\
\hline
\end{tabular}

Notes: all models were adjusted for age, sex, education, income, living arrangements, self-rated health, and chronic disease. ${ }^{*} p<0.05 ;{ }^{* *} p<0.01 ;{ }^{* * *} p<0.001$ Model 1: association between only PASE quantity and depression

Model 2: association between only patterns of PA and depression

Model 3: association between both PASE quantity, patterns of PA and depression 
patterns of PA provided information above and beyond PASE score alone (PASE scores, $\mathrm{r}^{2}=0.169$; patterns of PA, $r^{2}=0.178$; both, $r^{2}=0.182$ ).

Controlling for sociodemographic and health-related characteristics, PASE scores were negatively associated with depression. Older adults who had higher PASE scores (standardized) were less likely to be depressed $(O R=0.78,95 \% \mathrm{CI}=0.64-0.94)$. Patterns of PA also showed significant association with depression; compared with older adults who were domestic types, the athletic types were less likely to report depression $(O R=0.35,95 \% \mathrm{CI}=0.17-0.71)$, while the gardening/caring types were more likely to report depression $(O R=1.55,95 \% \mathrm{CI}=1.06-2.26)$. There were no significant differences in depression between the domestic types and the walkers.

\section{The joint influence of levels and patterns of PA on depression}

Figure 3 presents the multivariate-adjusted odds ratio of depression according to levels of PA (PASE quartile: $0-25 \%, 25-50 \%, 50-75 \%, 75-100 \%)$ and patterns of PA (domestic types, athletic types, gardening/caring types, and walkers). Results were adjusted for age, gender, education, income, marital status, living arrangements, and physical health status. Older adults who were the most active (PASE quartile: $75-100 \%$ ) and the athletic types had the strongest significant association with depression $(O R=0.19,95 \%$ CI: 0.06-0.65), followed by those who were the most active (PASE quartile: $75-100 \%$ ) and the walkers $(O R=0.28,95 \%$ CI: 0.14-0.57) when compared with older adults with the least activity (PASE quartile: $0-25 \%)$ and domestic types. Older adults who were the most active (PASE quartile: 75-100\%) and the gardening/caring types were most likely tend to be depressed $(O R=1.52$, 95\% CI: 0.94-2.46).

\section{Discussion}

Our study identified four patterns of PA in communitydwelling older adults in Shanghai, China, including domestic types, athletic types, gardening/caring types, and walkers. Our study supported patterns of PA provide information above and beyond PASE score alone. Patterns of PA is a multidimensional construct consisting of types, intensity, frequency, duration and volume, which can help to understand the relationship with depression comprehensively than just by a single dimensional analysis such as PASE quantity. Significantly, patterns of physical activity appeared to be associated with depression, because independent of the total amount of PA, athletic types seemed to have the strongest association with lower risk of depression. Gardening/caring types tended

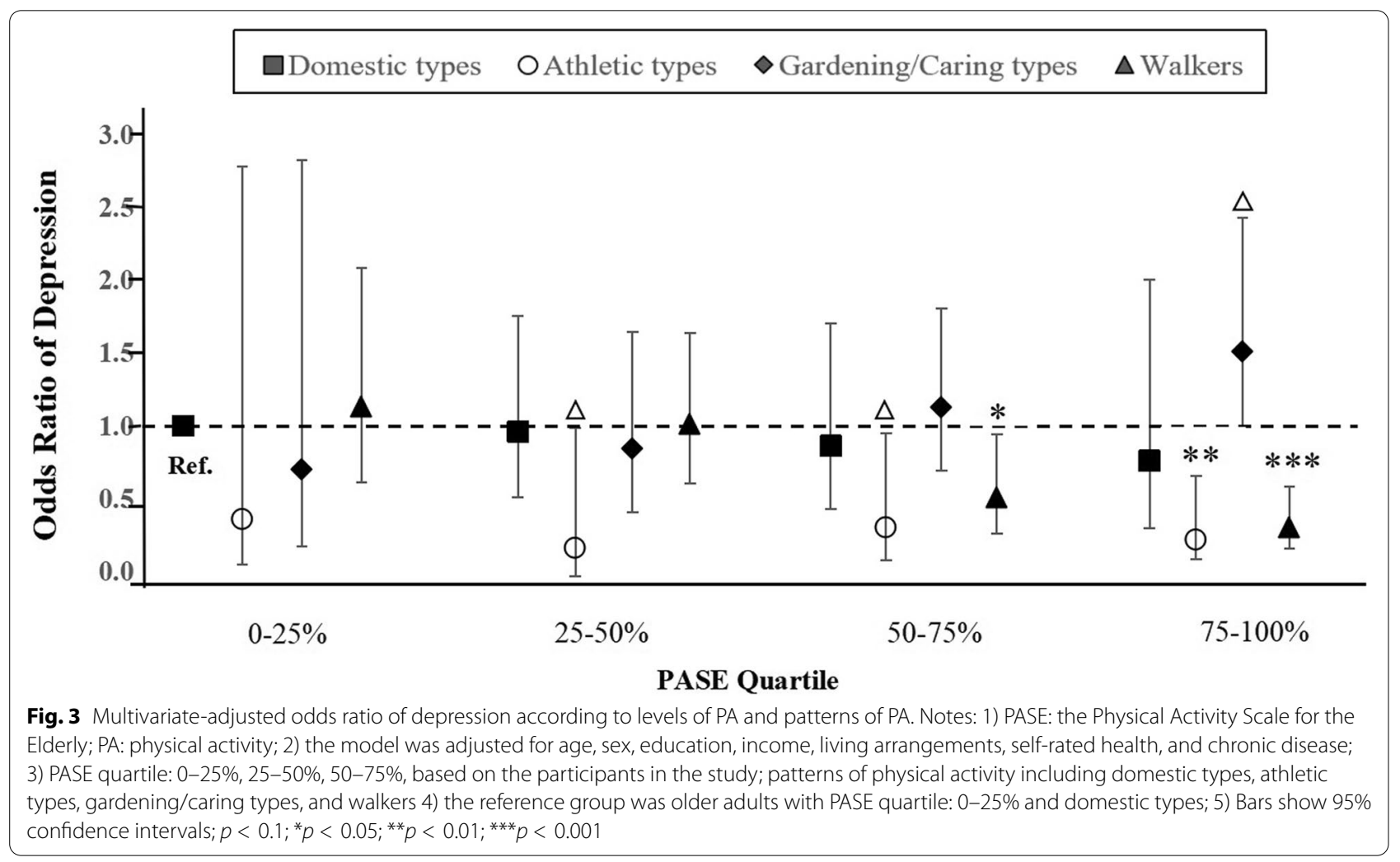


to be associated with a higher risk for depression in subjects with the highest level of activity (PASE quartile: 75-100\%).

To our knowledge, this is the first study that used a latent class approach to identify patterns of PA and explore their association with depression in Chinese community-dwelling older adults. Our results share several similarities with the findings of Mooney et al. and Joshi et al. [22, 35]. Mooney et al. [35] identified five patterns of physical activity in New York City residents aged 65-75: least active, walkers, athletic types, domestic/gardening athletic types, and domestic/gardening types. A different pattern, gardening/caring type was identified in our study might due to the specific cultural contexts in China. "Raising sons for old age" is a well-known Chinese saying. The young-old children take the primary responsibility of caring for their old-old parents [43]. Besides, grandparents commonly prefer to take care of grandchildren due to cultural traditions in East Asian countries [44].

Our findings supported a significant association between quantity of PA, patterns of PA and depression among community-dwelling older adults. When examining the joint effects of the quantity of physical activity and patterns of physical activity on depression risk, we found that a significant reduction in odds ratio was found only in the highest PA level, indicating that a doseresponse relationship might exist. Previous research showed different effects of various dose of PA on depression, for example, a larger treatment dose of exercise might result in a great improvement in depressive symptoms [45, 46]; and light-intensity PA showed a favorable effect on depression prevention, but moderate-to-vigorous PA was not $[47,48]$. Although we know little about the optimal amount of PA activity needed to reduce the risk of depression [49], our findings suggested that different patterns of PA might have different dose-response relationships with depression and that such differences were significant in the highest level of PA.

It is interesting to note that gardening/caring types tended to associate with a higher risk of depression, especially in older adults with the highest level of activities. These findings are in line with some previous studies that found caregivers were associated with higher risk of depression $[50,51]$. Although caring may provide the opportunity to experience the benefits of caring [52]; however, increased hours spent caring was associated with depression, as increasing caring time may decrease time for doing more pleasurable activities [53, 54]. This may explain why an adverse effect of caring on depression was observed in the highest level of PA. AndradeGómez et al. [55] pointed out that walking or playing sports might be more effective in reducing the risk of depression than domestic work, such as rearing children or gardening. This further emphasized the importance of inquiring about the underlying mechanisms regarding different patterns of PA and depression.

From a physiological perspective, physical activities such as walking or playing sports may cause immune system changes by reducing some inflammatory markers [56]. It was also found that monoamines and endorphins increased after exercise $[57,58]$. From psychosocial perspectives, PA may increase social interaction and enhance feelings of enjoyment, self-worth, and selfesteem [55]. Those physiological and psychosocial factors resulted in a lower risk of depression [55-58]. In line with previous studies [59-61], our findings support the benefits of exercise (walking and athletic activity) on depression. Our study suggested that domestic activities cannot replace exercise for depression prevention and that we must pay more attention to the older adults who are highly engaged in gardening and caring activities. Gardening or caring may be routine, obligatory, or repetitive [54], and with an increased level of such type of activities, pleasurable feelings may become reduced and physical exhaustion may occur [62]. It seems that both psychosocial experience and levels of PA do matter for depression. Further research is warranted to explore how different patterns and quantity of PA influence depression physiologically and psychologically.

\section{Strengths and limitations}

One of the main strengths of this study was the use of LCA to identify patterns of PA among Chinese community-dwelling older adults, solving the problem that different types of PA may be too highly correlated to be compared directly. The results of LCA can be used to direct the development of individualized interventions for the Chinese community-dwelling older adults. Community-based service and support program can be developed targeting to older adults with specific patterns of PA. For example, to provide respite care service to older adults who are highly engaged in gardening/caring for others. Further, we used population-based data to examine the joint effects of levels and patterns of PA on depression, which expanded our understanding of the associations among doses and patterns of PA and the likelihood of depression.

Several limitations should be noted. First, this study had a cross-sectional design, which precludes inference on causality. Second, data on PA were self-reported and thus could be prone to recall bias. Levels of PA (PASE quartile) were only based on our participants. Third, although we controlled for important confounders, there could still be bias due to unmeasured confounding variables, for example, we only used the presence of disease, 
but did not consider some specific diseases. Fourth, our sample was from only one district of Shanghai, China; thus, further study is warranted to replicate our results.

\section{Conclusion}

In conclusion, we found that both the quantity and patterns of physical activity are associated with depressive symptoms among community-dwelling older adults. Our findings suggested two important features to guide the development of interventions. First, population-level intervention should encourage community-dwelling older adults to increase their quantity of PA to reduce the risk of depression. Athletics and walkers are recommended. Second, to individually develop tailored interventions, more attention should be paid to older adults who are highly engaged in gardening/caring for others.

\section{Supplementary Information}

The online version contains supplementary material available at https://doi. org/10.1186/s12877-021-02537-8.

\section{Additional file 1.}

\section{Acknowledgments}

We would like to thank the Xinzhuang Town Community Management Office for assistance with participant recruitment. We are grateful to staff members of Shanghai Center for Disease Control and Prevention (SCDC), Xinzhuang Community Health Service Center of Minhang District, Medicine-Mental Health Center of Minhang District for their cooperation in the success of this study.

\section{Authors' contributions}

$Y L, X L, Y J, Y W$ participated in the design of the study, contributed to data collection, data analysis and interpretation of results; $Y Y, Y H$ participated in the design of the study; TY, ML, YR contributed to data collection and data analysis. All authors contributed to the manuscript writing. All authors have read and approved the final version of the manuscript, and agree with the order of authorship.

\section{Funding}

This work was support by the National Key Research and Development Project (2017YFC1310504), the National Natural Science Foundation of China (71673055), Tianqiao and Chrissy Chen for Translational Research (No.201801), Shanghai Municipal Science and Technology Major Project (No.2018SHZDZX01), Shanghai Leading Academic Discipline Project of Public Health (GWV-10.1-XK18), and ZJLab. The funder had no role in study design, data collection and analysis, decision to publish or preparation of the manuscript.

\section{Availability of data and materials}

Our data may not be shared directly, because it is our teamwork; informed consent should be attained from all the team members. Our data or material may be available after contacting the corresponding authors.

\section{Declarations}

\section{Ethics approval and consent to participate}

This research protocol was approved by the Ethical Review Board of Fudan University (reference number: IRB\#TYSQ 2019-2-03), and informed written consent was obtained before data collection. All methods were performed in accordance with the relevant guidelines and regulations.
Consent for publication

Not applicable.

\section{Competing interests}

The authors declare that they have no competing interests.

\section{Author details}

${ }^{1}$ School of Nursing, Fudan University, Shanghai 200032, China. ${ }^{2}$ Fudan University School of Public Health, Shanghai 200032, China. ${ }^{3}$ Shanghai Center for Disease Control and Prevention, Shanghai 200336, China. ${ }^{4}$ Shanghai Center for Clinical Laboratory, Shanghai 200126, China. ${ }^{5}$ Department of Geriatrics, Huashan Hospital Fudan University, Shanghai 200040, China. ${ }^{6}$ TianQiao and Chrissy Chen Institute Clinic Translational Research Center, Shanghai 200040, China. ${ }^{7}$ Shanghai Medicine-Mental Health Center of Minhang District, 130 DongAn Road, Shanghai 200032, China. ${ }^{8}$ Minhang Branch, School of Public Health, Fudan University, 130 DongAn Road, Shanghai 200032, China. ${ }^{9}$ Key Laboratory of Health Technology Assessment, National Health and Family Planning Commission of the People's Republic of China, Fudan University, 130 DongAn Road, Shanghai 200032, China.

Received: 21 December 2020 Accepted: 7 October 2021

Published online: 21 October 2021

\section{References}

1. Beekman AT, Copeland JR, Prince MJ. Review of community prevalence of depression in later life. Br J Psychiatry. 1999;174:307-11.

2. Djernes JK. Prevalence and predictors of depression in populations of elderly: a review. Acta Psychiatr Scand. 2006;113:372-87.

3. Alexopoulos GS. Depression in the elderly. Lancet. 2005;365:1961-70.

4. Wilmot EG, Edwardson CL, Achana FA, Davies MJ, Gorely T, Gray L, et al. Sedentary time in adults ah8nd the association with diabetes, cardiovascular disease and death: systematic review and meta-analysis. Diabetologia. 2012;55:2895-905.

5. Saz P, Dewey ME. Depression, depressive symptoms and mortality in persons aged 65 and over living in the community: a systematic review of the literature. Int J Geriatr Psych. 2001;16:622-30.

6. Global Burden of Disease 2015 DALYs and HALE Collaborators. Global, regional, and national disability-adjusted life-years (DALYS) for 315 diseases and injuries and healthy life expectancy (HALE), 1990-2015: A systematic analysis for the Global Burden of Disease Study 2015. Lancet. 2016; 388: 1603-1658.

7. Mobily KE, Rubenstein LM, Lemke JH, O'Hara MW, Wallace RB. Walking and depression in a cohort of older adults: the lowa $65+$ rural health study. J Aging Phys Act. 1996:4:119-35.

8. Morgan K, Bath PA. Customary physical activity and psychological wellbeing: a longitudinal study. Age Ageing. 1998;27(Suppl 3):35-40.

9. Lampinen P, Heikkinen RL, Ruoppila I. Changes in intensity of physical exercise as predictors of depressive symptoms among older adults: an eight-year follow-up. Prev Med. 2000;30:371-80.

10. Kritz-Silverstein D, Barrett-Connor E, Corbeau C. Cross-sectional and prospective study of exercise and depressed mood in the elderly the rancho Bernardo study. Am J Epidemiol. 2001;153:596-603.

11. Lee C, Russell A. Effects of physical activity on emotional wellbeing among older Australian women: cross-sectional and longitudinal analyses. J Psychosom Res. 2003;54:155-60.

12. Almeida OP, Norman P, Hankey G, Jamrozik K, Flicker L. Successful mental health aging: results from a longitudinal study of older Australian men. Am J Geriatr Psychiatry. 2006;14:27-35.

13. McHugh JE, Lawlor BA. Exercise and social support are associated with psychological distress outcomes in a population of community-dwelling older adults. J Health Psychol. 2012;17:833-44.

14. Cunningham C, O'Sullivan R, Caserotti P, Tully MA. Consequences of physical inactivity in older adults: a systematic review of reviews and meta-analyses. Scand J Med Sci Sports 2020; 30(5):816-827.

15. Falck RS, McDonald SM, Beets MW, Brazendale K, Liu-Ambrose T. Measurement of physical activity in older adult interventions: a systematic review. Br J Sports Med. 2016;50(8):464-87. 
16. Moore KA, Babyak MA, Wood CE, Napolitano MA, Khatri P, Craighead WE, et al. The association between physical activity and depression in older depressed adults. J Aging Phys Activ. 1999;7(1):55-61.

17. Strawbridge WJ, Deleger S, Roberts RE, Kaplan GA. Physical activity reduces the risk of subsequent depression for older adults. Am J Epidemiol. 2002;156(4):328-34.

18. Wang S, Ma W, Wang S-M, Yi X. A cross sectional examination of the relation between depression and frequency of leisure time physical exercise among the elderly in Jinan, China. Int J Environ Res Public Health. 2018;15(9):2041.

19. Kanamori S, Takamiya T, Inoue S, Kai Y, Tsuji T, Kondo K. Frequency and pattern of exercise and depression after two years in older Japanese adults: the JAGES longitudinal study. Sci Rep. 2018;8.

20. Jung S, Lee S, Lee S, Bae S, Imaoka M, Harada K, et al. Relationship between physical activity levels and depressive symptoms in community-dwelling older Japanese adults. Geriatr Gerontol Int. 2018;18:421-7.

21. Bishwajit G, O'Leary DP, Ghosh S, Yaya S, Tang SF, Feng ZC. Physical inactivity and self-reported depression among middle- and older-aged population in South Asia: world health survey. BMC Geriatr. 2017;17:8.

22. Joshi S, Mooney SJ, Kennedy GJ, Benjamin EO, Ompad D, Rundle AG, et al. Beyond METs: types of physical activity and depression among older adults. Age Ageing. 2016;45:103-9.

23. Whitehead BR, Blaxton JM. Daily well-being benefits of physical activity in older adults: does time or type matter? Gerontologist. 2017;57(6):1062-71.

24. Teychenne M, Ball K, Salmon J. Physical activity and likelihood of depression in adults: a review. Prev Med. 2008:46:397-411.

25. Olanrewaju O, Kelly S, Cowan A, Brayne C, Lafortune L. Physical activity in community dwelling older people: a systematic review of reviews of interventions and context. PLoS One. 2016;11(12):e0168614.

26. Chang Y-C, Lu M-C, Hu IH, Wu W-Cl HSC. Effects of different amounts of exercise on preventing depressive symptoms in communitydwelling older adults: a prospective cohort study in Taiwan. BMJ Open. 2017;7(4):e014256.

27. Huh J, Riggs NR, Spruijt-Metz D, Chou CP, Huang Z, Pentz M. Identifying patterns of eating and physical activity in children: a latent class analysis of obesity risk. Obesity. 2011;19(3):652-8.

28. Iannotti RJ, Wang J. Patterns of physical activity, sedentary behavior, and diet in U.S. adolescents. J Adolesc Health. 2013;53(2):280-6.

29. Silverwood RJ, Nitsch D, Pierce M, Kuh D, Mishra GD. Characterizing longitudinal patterns of physical activity in mid adulthood using latent class analysis: results from a prospective cohort study. Am J Epidemiol. 2011;174:1406-15.

30. Washburn RA, Smith KW, Jette AM, Janney CA. The physical activity scale for the elderly (PASE): development and evaluation. J Clin Epidemiol. 1993:46(2):153-62.

31. Washburn RA, McAuley E, Katula J, Mihalko SL, Boileau RA. The physical activity scale for the elderly (PASE): evidence for validity. J Clin Epidemiol. 1999;52:643-51.

32. Harada ND, Chiu V, King AC, Stewart AL. An evaluation of three self-report physical activity instruments for older adults. Med Sci Sports Exerc. 2001;33(6):962-70.

33. Bonnefoy M, Normand S, Pachiaudi C, Lacour JR, Laville M, Kostka T. Simultaneous validation of ten physical activity questionnaires in older men: a doubly labeled water study. J Am Geriatr Soc. 2001;49(1):28-35.

34. Vaughan K, Miller WC. Validity and reliability of the Chinese translation of the physical activity scale for the elderly (PASE). Disabil Rehabil. 2013;35(3):191-7.

35. Mooney SJ, Joshi S, Cerdá M, Quinn JW, Beard JR, Kennedy GJ, et al. Patterns of physical activity among older adults in new York City: a latent class approach. Am J Prev Med. 2015;49(3):e13-22.

36. Yesavage JA, Brink TL, Rose TL, Lum OL, Huang V, Adey M, et al. Development and validation of a geriatric depression screening scale: a preliminary report. J Psychiatr Res. 1982;17(1):37-49.

37. Brink TL, Md JAY, Md OL, Md PH, Ba MA, Rose TL. Screening tests for geriatric depression. Clin Gerontol. 2008;1 (1):37-43.

38. Chan AC. Clinical validation of the geriatric depression scale (GDS) Chinese version. J Aging Health. 1996;8(2):238-53.

39. Carragher N, Adamson G, Bunting B, McCann S. Subtypes of depression in a nationally representative sample. J Affect Disord. 2009;113(1-2):88-99.
40. Nylund KL, Asparouhov T, Muthén BO. Deciding on the number of classes in latent class analysis and growth mixture modeling: a Monte Carlo simulation study. Struct Equ Modeling. 2007;14(4):535-69.

41. Celeux G, Soromenho G. An entropy criterion for assessing the number of clusters in a mixture model. J Classif. 1996;13(2):195-212.

42. Petras H, Masyn K. General growth mixture analysis with antecedents and consequences of change. In: Piquero R, Weisburd D, editors. Handbook of quantitative criminology. New York, NY: Springer; 2010. p. 69-100.

43. Liu J, Guo M, Bern-Klug M. Economic stress among adult-child caregivers of the oldest old in China: the importance of contextual factors. J Cross Cult Gerontol. 2013;28:465-79.

44. Lou, V. W. Q., \& Chi, I. Grandparenting roles and functions. In K. K. Mehta \& L. L. Thang (Eds.), Experiencing grandparenthood: An Asian perspective. Dordrecht, the Netherlands: Springer. 2012; p 47-59.

45. Cheng S, Chow PK, Yu ES, Chan AM. Leisure activities alleviate depressive symptoms in nursing home residents with very mild or mild dementia. AM J Geriat Psychiat. 2012;20(10):904-8.

46. Tsang HW, Tsang WW, Jones AY, Fung KM, Chan AH, Chan EP, et al. Psychophysical and neurophysiological effects of qigong on depressed elders with chronic illness. Aging Ment Health. 2013;17(3):336-48.

47. Lanza ST, Rhoades BL. Latent class analysis: an alternative perspective on subgroup analysis in prevention and treatment. Prev Sci. 2013;14(2):157-68.

48. Yasunage A, Shibata A, Ishii K, Koohsari MJ, Oka K. Cross-sectional associations of sedentary behaviour and physical activity on depression in Japanese older adults: an isotemporal substitution approach. BMJ Open. 2018;8:e022282.

49. Hallgren M, Herring MP, Owen N, Dunstan D, Ekblom O, Helgadottir B, et al. Exercise, physical activity, and sedentary behavior in the treatment of depression: broadening the scientific perspectives and clinical opportunities. Front Psychiatry. 2016;7:36.

50. Koyanagi A, DeVylder JE, Stubbs B, Carvalho AF, Veronese N, Haro $\mathrm{JM}$, et al. Depression, sleep problems, and perceived stress among informal caregivers in 58 low-, middle-, and high-income countries: a cross-sectional analysis of community-based surveys. J Psychiatr Res. 2018;96:115-23.

51. Choi KS, Stewart R, Dewey M. Participation in productive activities and depression among older Europeans: survey of health, ageing and retirement in Europe (SHARE). Int J Geriatr Psychiatry. 2013;28:1157-65.

52. Pinquart $M$, Sorensen $S$. Associations of caregiver stressors and uplifts with subjective well-being and depressive mood: a meta-analytic comparison. Aging Ment Health. 2004;8:438-49.

53. Loi SM, Dow B, Moore K, Hill K, Russell M, Cyarto E, et al. Factors associated with depression in older carers. Int J Geriatr Psych. 2016;31:294-301.

54. Chen L, Stevinson C, Ku PW, Chang YK, Chu DC. Relationships of leisuretime and non-leisure-time physical activity with depressive symptoms: a population based study of Taiwanese older adults. Int J Behav Nutr Phys Act. 2012;9:28-37.

55. Andrade-Gómez E, Martínez-Gómez D, Rodríguez-Artalejo F, GarcíaEsquinas E. Sedentary behaviors, physical activity, and changes in depression and psychological distress symptoms in older adults. Depress Anxiety. 2018;35:884-97.

56. Hamer M, Molloy GJ. deOliveira C, Demakakos P. leisure time physical activity, risk of depressive symptoms, and inflammatory mediators: the English longitudinal study of ageing. Psychoneuroendocrinology. 2009;34:1050-5.

57. Phillips WT, Kiernan M, King AC. The effects of physical activity on physical and psychological health. In: Baum A, Revenson TA, Singer JE, editors. Handbook of health psychology. London: Lawrence Erlbaum Associates; 2001. p. 627-57.

58. Thornen P, Floras JS, Hoffman P. Endorphins and exercise: physiological mechanisms and clinical implications. Med Sci Sports Exerc. 1990;22:417-28.

59. Taylor-Piliae RE, Haskell WL, Waters CM, Froelicher ES. Change in perceived psychosocial status following a 12-week tai Chi exercise programme. J Adv Nurs. 2006;54:313-29.

60. Kohut ML, McCann DA, Russell DW, Konopka DN, Cunnick JE, Franke WD, et al. Aerobic exercise, but not flexibility/resistance exercise, reduces serum IL-18, CRP, and IL-6 independent of beta-blockers, BMI, and psychosocial factors in older adults. Brain Behav Immun. 2006;20:201-9. 
61. Gerber M, Brand S, Herrmann C, Colledge F, Holsboer-Trachsler E, Pühse $U$. Increased objectively assessed vigorous-intensity exercise is associated with reduced stress, increased mental health and good objective and subjective sleep in young adults. Physiol Behav. 2014;135:17-24.

62. Pickett K, Yardly L, KendrickT. Physical activity and depression: a multiple mediation analysis. Ment Health Phys Act. 2012;5:125-34.

\section{Publisher's Note}

Springer Nature remains neutral with regard to jurisdictional claims in published maps and institutional affiliations.
Ready to submit your research? Choose BMC and benefit from:

- fast, convenient online submission

- thorough peer review by experienced researchers in your field

- rapid publication on acceptance

- support for research data, including large and complex data types

- gold Open Access which fosters wider collaboration and increased citations

- maximum visibility for your research: over $100 \mathrm{M}$ website views per year

At BMC, research is always in progress.

Learn more biomedcentral.com/submissions 